\title{
Long-Range Coupling Affects Entanglement Dynamics
}

\author{
Three new studies predict the measurement-induced phase-transition \\ behavior for quantum systems that have long-range coupling between \\ their qubits.
}

By Shenglong Xu

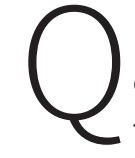

uantum computers derive their power from the complex entanglement structure that "builds" between their qubits over time. But reading out information from such a computer requires measuring the state of individual qubits, a process that can impact the system's entanglement as it forces each qubit into a particular state,

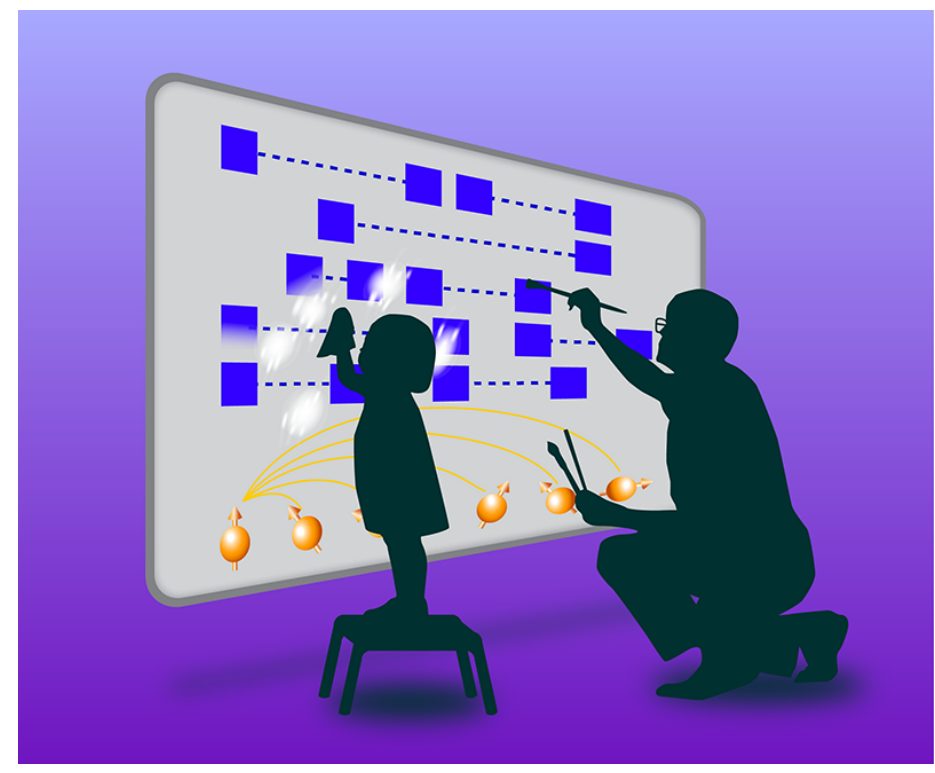

Figure 1: Schematic diagram showing the competition between entanglement building and entanglement erasing in quantum systems with long-range coupling between their qubits. The rivalry between the processes can be likened to an artist trying to draw a picture (entanglement building) while a child simultaneously rubs it out (entanglement erasing).

Credit: APS/Carin Cain disentangling it from the others.

Disentangling one qubit from a quantum computer does not significantly affect that computer's overall entanglement structure, but measuring more and more qubits will eventually "erase" the entanglement, turning the system classical. That said, interesting quantum things can happen when repetitive measurements of randomly selected qubits occur in an evolving entangled system [1-3]. Three teams of researchers now theoretically investigate the outcome of the competition between "entanglement erasing" and "entanglement building" in quantum many-body systems that have long-range coupling between their qubits (Fig. 1) [4-6]. Their findings reveal rich dynamical behaviors and complex phase transitions that depend on two parameters: the rate at which qubits are measured and the spatial extent of the coupling. The results indicate that it should be possible to control the entanglement structure between qubits by tuning these two parameters, which could lead to new dynamical phases in quantum many-body systems.

The entanglement structure of a quantum many-body system can be quantified through the system's bipartite entanglement entropy-a parameter that quantifies the entanglement between two sides of a system, for example, the left and right. When no measurements are made, this entropy typically grows linearly over time before saturating at a value that is proportional to the system's volume. However, theory has shown that the saturation value is lowered by repetitive measurements of random qubits in a quantum many-body system. For example, in a system consisting of a hybrid quantum circuit of qubits with short-range coupling, 
researchers predict that increasing the rate of measurement causes the entropy to saturate at a lower value that is independent of the system size [1-3]. A measurement-induced phase transition (MIP) occurs between these two behaviors in the form of a vanishing entanglement-entropy density in the thermodynamic limit.

Besides systems with short-range coupling, MIPs also occur in quantum many-body systems with long-range couplings between qubits, such as arrays of Rydberg atoms or trapped ions. Long-range couplings fall off slowly with distance as $1 / r^{\alpha}$, where $r$ is the separation between the qubits and $\alpha$ is an exponent controlling the coupling's decay. This coupling is known to enhance the entanglement-building power of a quantum many-body system, as it enables direct entanglement between distant qubits. It was unknown, however, how measurements (and entanglement erasing) would compete with this enhanced entanglement building. In other words, how does long-range coupling affect a system's MIP? Three teams have now independently explored this question [4-6]. The teams study this problem in different quantum many-body systems and use different approaches, but they all find that MIPs survive for large $\alpha$. They also predict that the systems that they study will exhibit rich dynamical behaviors as $\alpha$ decreases.

Two of the teams considered the same platform: a quantum Hamiltonian system of fermions with long-range hopping. These fermions do not directly interact with each other, but they can hop between two spatially distant lattice sites. This hopping acts as the coupling-like behavior explored in other quantum many-body systems [4-6]. Previous research looked at free fermions with short-ranged hopping, finding that the MIP occurs when the saturation value goes from being proportional to the logarithm of the system's size to being independent of the system's size [7]. In the new studies, performed by Thomas Müller and colleagues from the University of Cologne, Germany, and Takaaki Minato from Keio University, Japan, and colleagues, the same short-ranged MIP behavior is found for long-range hopping, but only when the decay exponent is large: $\alpha>1.5$.

Müller and colleagues developed a model to understand the origins of this critical value of $\alpha$ [6]. When $1<\alpha<1.5$, they find that long-range interactions induce no MIP but instead cause the system to sit in a phase where the entanglement entropy grows and saturates at a value that goes as $L^{3 / 2-\alpha}$, where $L$ is the system's volume. Minato and colleagues also explored different values of $\alpha$, deriving for it a lower bound for a MIP to occur that relates to a system's entanglement entropy generation rate [4]. For a 1D system of free fermions with long-range hopping, the team finds a lower bound of $\alpha=1.5$, which agrees with the results of Müller and colleagues. By accounting for long-range density-density interactions, Minato and colleagues further generalized their approach to the case where the fermions also interact. They find that the interactions increase the lower bound on an MIP's appearance to $\alpha=2$.

A third team, which was made up of Maxwell Block and colleagues from the University of California, Berkeley, looked instead at a quantum-circuit-model platform. Mapping the circuit model to a long-range Ising model and then carrying out numerical simulations of so-called Clifford circuits, they find that MIP behavior occurred for their model for values of $\alpha>3$.

Block and his colleagues studied MIPs for values of $\alpha$ from above 3 down to 0 , where the coupling becomes all-to-all-meaning the coupling is the same between all qubits, regardless of distance. They find a sequence of dynamical phases and transitions for different values of $\alpha$ that are distinct from the MIP found in the same system with short-range coupling. In particular, when $\alpha<1$, the team observes that the saturating entanglement entropy is always proportional to the system size, but that measurement induces a phase transition in the dynamics of the system's entanglement structure $[8,9]$.

Controlling the entanglement structure of a quantum many-body system has fundamental theoretical interest as well as practical importance. These three new results suggest that it is possible to alter the entanglement structure of a quantum many-body system's steady state by tuning the rate at which measurements are made and the coupling range of the system's qubits. The next step for researchers will be characterizing in more depth the unique entanglement structures that the three teams find and then harnessing those structures for quantum many-body applications, such as encoding, teleporting and decoding quantum information, and engineering nonequilibrium phase of matter. Recently, MIPs have been predicted and experimentally observed for a quantum computer made of trapped ions [10]. It would be interesting to design protocols to experimentally observe the predicted 
transitions and phases as a function of the coupling range. Another interesting direction is to study how this tunable MIP behavior can be affected by quenched disorder, which tends to slow down a quantum system's dynamics.

Shenglong Xu: Texas A\&M University, College Station, TX, USA

\section{REFERENCES}

1. B. Skinner et al., "Measurement-induced phase transitions in the dynamics of entanglement," Phys. Rev. X 9, 031009 (2019).

2. Y. Li et al., "Quantum zeno effect and the many-body entanglement transition," Phys. Rev. B 98, 205136 (2018).

3. A. Chan et al., "Unitary-projective entanglement dynamics," Phys. Rev. B 99, 224307 (2019).

4. T. Minato et al., "Fate of measurement-induced phase transition in long-range interactions," Phys. Rev. Lett. 128, 010603 (2022).
5. M. Block et al., "Measurement-induced transition in long-range interacting quantum circuits," Phys. Rev. Lett. 128, 010604 (2022).

6. T. Müller et al., "Measurement-induced dark state phase transitions in long-ranged fermion systems," Phys. Rev. Lett. 128, 010605 (2022).

7. O. Alberton et al., "Entanglement transition in a monitored free-fermion chain: From extended criticality to area law," Phys. Rev. Lett. 126, 170602 (2021).

8. M. J. Gullans and D. A. Huse, "Dynamical purification phase transition induced by quantum measurements," Phys. Rev. $X$ 10, 041020 (2020).

9. S. Vijay, "Measurement-driven phase transition within a volume-law entangled phase," arXiv:2005.03052.

10. C. Noel et al., "Observation of measurement-induced quantum phases in a trapped-ion quantum computer," arXiv:2106.05881. 